\title{
Lipid-Producing Ciliochoroidal Melanoma with Expression of HMG-CoA Reductase
}

\author{
David Van Lya,b Duo Wang ${ }^{a}$ Robert Max Conwayc, d Michael Giblin ${ }^{d}$ \\ Sharron Liang ${ }^{a}$ Robyn Lukeis ${ }^{a}$ Li-Anne Lim ${ }^{d}$ Luke Hesson $^{e}, f$ \\ Svetlana Cherepanoffa, $\mathrm{g}$
}

${ }^{a}$ Anatomical Pathology, SydPath, St Vincent's Hospital, Sydney, NSW, Australia; ${ }^{b}$ School of Medicine, The University of Notre Dame Australia, Sydney, NSW, Australia; 'Save Sight Institute, The University of Sydney, Sydney, NSW, Australia; ' Sydney Eye Hospital, Sydney, NSW, Australia; ${ }^{e}$ Kinghorn Centre for Clinical Genomics, Garvan Institute of Medical Research, Darlinghurst, NSW, Australia; ${ }^{f}$ Faculty of Medicine, Prince of Wales Clinical

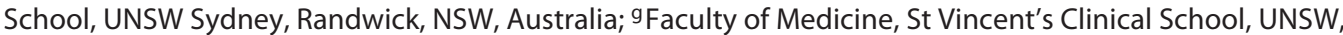
Darlinghurst, NSW, Australia

\section{Established Facts}

- Lipid synthesis and metabolism can be key drivers of tumour survival.

- Lipid production has recently been described in uveal melanoma (UM).

- The mevalonate pathway is important in lipid metabolism, and HMG-CoA reductase is the rate-controlling enzyme; it is also the target of statins.

\section{Novel Insights}

- Lipid-producing UM may express HMG-CoA reductase, providing evidence for mevalonate pathway activity and cholesterol synthesis.

- Lipid-producing UM can have a high-risk molecular genetic signature.

- These tumour characteristics suggest novel targets, particularly inhibitors of the mevalonate pathway, as potential therapies for this unusual phenotype.

\section{Keywords}

Uveal melanoma - Lipids · Statin · Cholesterol · HMG-CoA reductase

\begin{abstract}
Uveal melanoma (UM) is the commonest primary intraocular malignancy in adults. There is limited published data on lipid production in UM. Here, we describe the clinical, histological, immunohistochemical, and molecular findings in a
\end{abstract}


ciliochoroidal melanoma with lipid production and expression of the enzyme HMG-CoA reductase. This case highlights an unusual UM tumour phenotype with a high-risk molecular metastatic profile and discusses tumour lipogenesis and activation of the mevalonate pathway as a potential therapeutic target in managing lipidised ciliochoroidal UM.

(c) 2020 S. Karger AG, Basel

\section{Introduction}

Uveal melanoma (UM) is the commonest intraocular malignancy in adults, with an incidence of 5.2 per million in the USA [1,2] and up to 8.6 per million in Europe [3]. Up to $50 \%$ of the patients develop metastases. Survival for metastatic UM remains extremely poor with no effective treatments. Lipid synthesis and metabolism are known to play an important role in tumour survival in cancer cell biology [4]. Lipid production in UM has received relatively little research attention $[5,6]$. In this report, we aim to add to this body of knowledge by describing the clinical, histological, immunohistochemical, molecular, and cytogenetic findings in a lipid-producing ciliochoroidal melanoma with a high metastatic risk. We examine tumour expression of HMG-CoA reductase and discuss mevalonate pathway inhibition as a possible therapeutic approach.

\section{Case Report}

A 39-year-old obese male patient with Type 2 diabetes mellitus and no background of cholesterol-lowering medication use presented with a 3-month history of blurry vision in the left eye. Full examination and B-scan ultrasonography revealed a superonasal ciliochoroidal melanoma with a base of approximately $16.0 \mathrm{~mm}$ and thickness $11.0 \mathrm{~mm}$ in the left eye with associated inferotemporal serous retinal detachment (Fig. 1a). The primary tumour was surgically managed by enucleation; however, it was not long before the patient developed metastases and was palliated. Macroscopically, the tumour had a minimally pigmented, yellow, and fatty cut surface with prominent vascular lakes (Fig. 1b, c). It measured 18 $\mathrm{mm}$ (base) $\times 12 \mathrm{~mm}$ (thickness) and extended from the pars plicata to within $3 \mathrm{~mm}$ of the optic disc.

On microscopy, the tumour had a nodular architecture (Fig. 1d) with vascular lakes and extravascular matrix loops forming networks. The tumour showed strong diffuse MART1 immunostaining (Fig. 1e) and loss of nuclear BAP1 expression (Fig. 1f). Most tumour cells were epithelioid, and many had foamy cytoplasm consisting of intracytoplasmic vacuoles (Fig. 1g). The vacuoles did not contain glycogen (negative on PAS special staining; Fig. 1h) and were confirmed to consist of lipid on adipophilin immunohistochemistry (Fig. 1i). Many of the lipid-laden tumour cells also showed strong cytoplasmic HMG CoA reductase expression (Fig. 1j).

Lipid-Producing Ciliochoroidal

Melanoma
Immunohistochemistry was performed using routine diagnostic primary antibodies (online suppl. Table S1; for all online suppl. material, see www.karger.com/doi/10.1159/000510393), proprietary consumables, and protocols based on the Ventana BenchMark Ultra platform (Roche Diagnostics, North Ryde, NSW, Australia).

Molecular cytogenetic CGH/SNP microarray was performed on fresh tumour material using the CytoScan $750 \mathrm{~K}$ assay (Applied Biosystems, ThermoFisher, North Ryde, NSW, Australia) according to the manufacturer's protocol, and DNA was extracted using the Gentra Puregene kit (Qiagen). Analysis of the array results was performed using the Chromosome Analysis Suite (ChAS; version 3.1) and Mayrhofer et al. [7]. Monosomy for whole chromosome 3 was identified, together with whole arm gain of $8 \mathrm{q}(5$ copies $)$ and loss of $8 \mathrm{p}$, consistent with 2 copies of an isochromosome $8 \mathrm{q}$. Additional abnormalities included whole arm deletion of $16 \mathrm{q}$, and sub-clonal segmental deletion $6 \mathrm{q} 16.3 \mathrm{q} 27$ ( $~ 50 \%$ of the cells) and gain 16 p11.2p13.3 ( $20 \%$ of the cells; Fig. $1 \mathrm{k}$ ).

To determine if there was a unique tumour mutational profile associated with this lipid-producing UM phenotype, DNA and RNA were extracted from formalin-fixed paraffin embedded tumour tissue and assayed by NGS using the Oncomine Comprehensive and Tumour Mutation Load (TML) Assay on the Ion GeneStudio S5 platform (ThermoFisher; online suppl. Table S2). Coding region missense and nonsense variants with a variant allele frequency $>0.05$ with $\geq 20$ variant reads and total coverage $\geq 400$ reads were identified using Torrent Suite (version 5.10.1) and Ion Reporter (version 5.10.5.0) software (ThermoFisher). RNA data was assessed for the presence of potential gene fusions. Pathogenicity and clinical actionability were determined using Qiagen Clinical Insights version 1.4 (Qiagen, Chadstone, VIC, Australia) in accordance with ACMG and AMP/ASCO/CAP guidelines (Table 1) $[8,9]$.

RNA sequencing passed quality metrics, but no gene fusion events were detected. Missense mutations were detected in 7 known tumour-associated genes (Table 2; online suppl. Table S3). The presence of a GNA11 mutation (c.626A > T p.[Gln209Leu]) confirmed the tumour's uveal melanocytic differentiation. Detection of a BAP1 mutation (c.203A > G p.[Asp68Gly]) was consistent a high-risk UM mutational signature $[10,11]$.

Fig. 1. a B-scan ultrasonography showing a ciliochoroidal melanoma of the left eye with a base of approximately $15.93 \mathrm{~mm}$ and a thickness of $11.69 \mathrm{~mm}$. b, c Macroscopic photography of the enucleated eye shows a ciliochoroidal tumour, a minimally pigmented, fatty cut surface, and conspicuous vascular lakes, measuring 18 $\mathrm{mm}$ (basal diameter) $\times 12.0 \mathrm{~mm}$ (height; d). H\&E stained section showing a tumour with nodular architecture and epithelioid cytomorphology, with (e) diffuse strong MART1 immunostaining and (f) loss of nuclear BAP1 expression. g Higher power $(\times 400) \mathrm{H} \& \mathrm{E}$ section showing intracytoplasmic vacuoles, which were negative on PAS special staining (h) but stained positively for adipophilin on immunohistochemistry (i). j HMG Co-A reductase staining is seen in the same vacuolated adipophilin+ tumour cells. $\mathbf{k} \mathrm{CGH} /$ SNP analysis of the tumour revealed multiple molecular abnormalities, including monosomy for chromosome 3 , gain of $8 \mathrm{q}$ (5 copies) with deletion of $8 p$. Subclonal deletion of $6 q$ and of $16 q$ were also detected.

(For figure see next page.)

Ocul Oncol Pathol 2020;6:416-421 

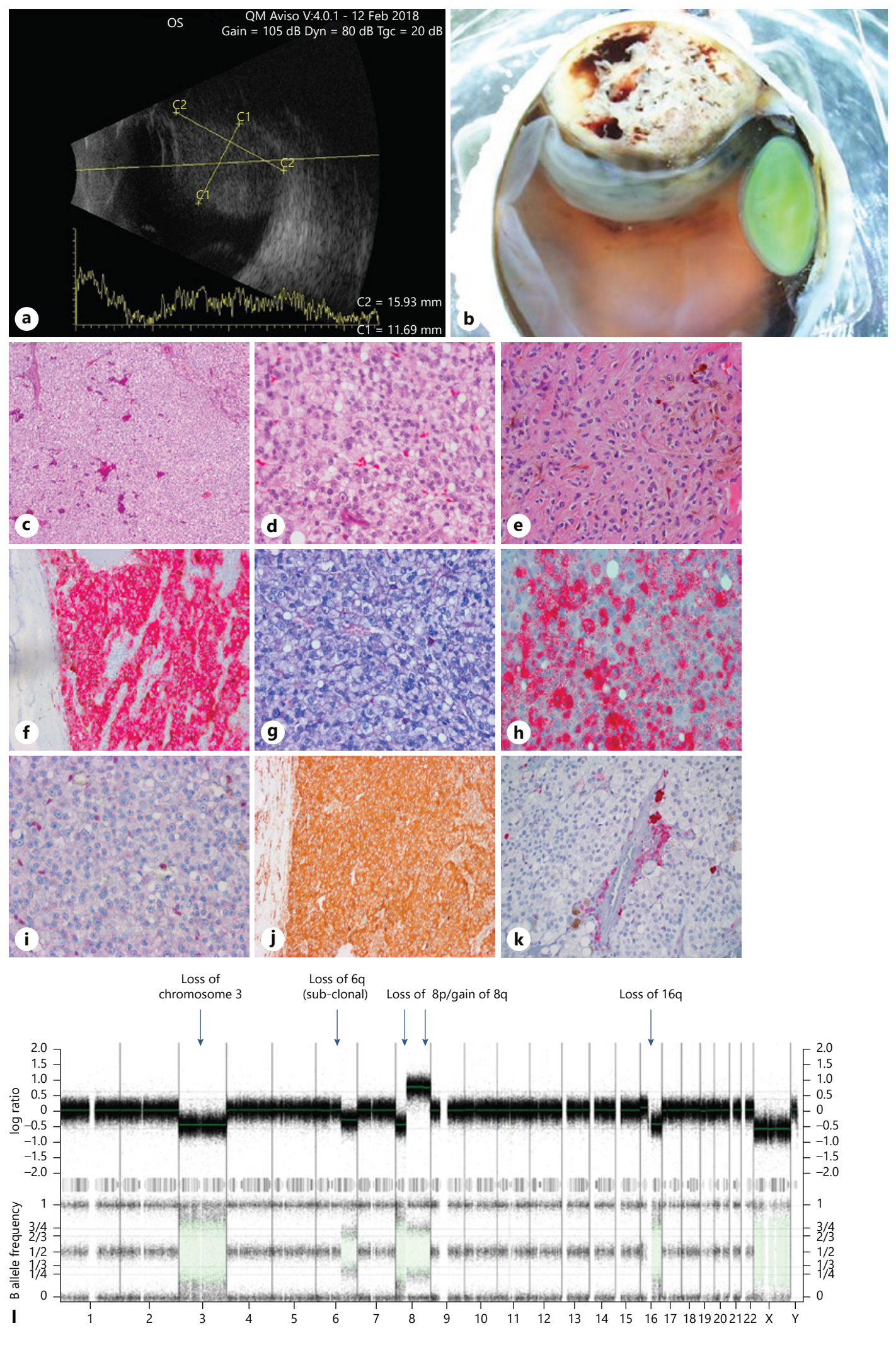
Table 1. Criteria to report clinically significant and pathological variants

\begin{tabular}{|c|c|c|}
\hline No. & Criterion & Description \\
\hline 1 & Sequencing quality metrics of the variant & $\begin{array}{l}\text { Is the variant truly present based on coverage and quality } \\
\text { scores? }\end{array}$ \\
\hline 2 & Presence in the COSMIC database & Has it been seen before in cancer? \\
\hline 3 & Absence from population databases & $\begin{array}{l}\text { Is the variant just a benign germline single-nucleotide } \\
\text { polymorphism? }\end{array}$ \\
\hline 4 & Evidence of clinical actionability and/or pathogenicity & $\begin{array}{l}\text { Is the variant linked to a therapy or clinical trial, and is there } \\
\text { evidence it causes loss or gain of gene function? }\end{array}$ \\
\hline
\end{tabular}

Adapted from international standards and guidelines for the interpretation of sequence variants $[8,9]$.

Table 2. Mutations identified in the primary case using the oncomine comprehensive and tumour mutation load assays

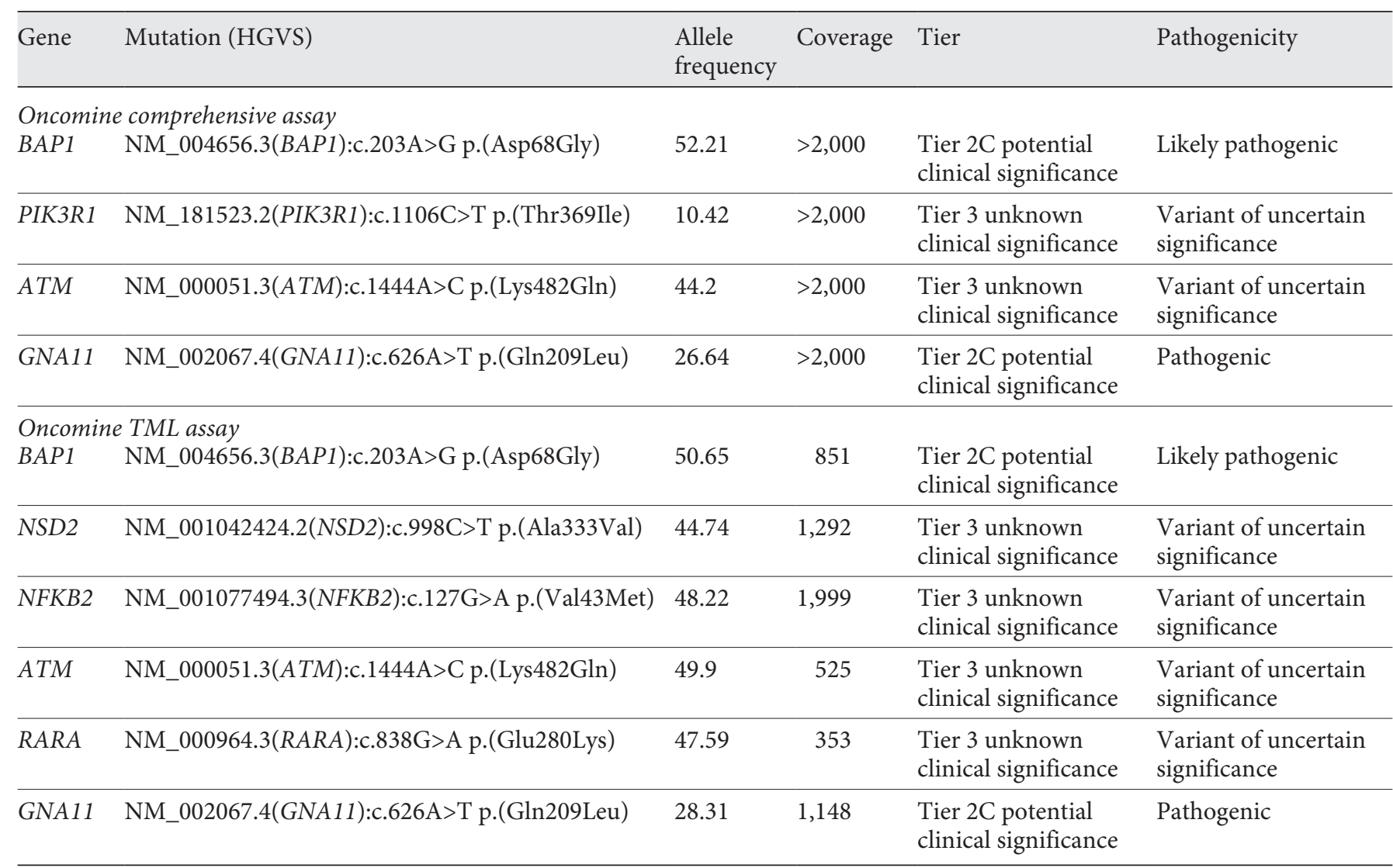

Tier refers to clinical actionability according to AMP/ASCO/CAP guidelines [9]. Pathogenicity was determined according to ACMG guidelines [8].

\section{Discussion and Conclusion}

It has been long recognized that chronic injury and degeneration due to ischaemia and inflammation can contribute to lipidisation of neoplastic cells [4]. Lipogenic pathways were later shown to be upregulated in proliferating cells [12]. Importantly, altered lipid production has been shown to be a feature of cutaneous melanoma 
[13], and high expression may be a prognostic factor associated with more advanced disease [14]. Recently, lipid production has also been demonstrated in a subset of $\mathrm{UM}$, but the drivers of this lipidic profile remain unclear $[5,6]$.

The current report describes expression of HMG-CoA reductase in a lipid-producing UM with high-risk molecular cytogenetic (combined monosomy 3 and high level $8 \mathrm{q}$ gain) $[15,16]$ and mutational signatures. HMG$\mathrm{CoA}$ reductase is the rate-controlling enzyme of the mevalonate lipid metabolic pathway, which plays crucial roles in oncogenesis and cancer cell stemness $[17,18]$. Enhanced cholesterol biosynthesis has been shown to play a role in prostate cancer development (reviewed by Hager et al. [19]), for example, and blocking the mevalonate pathway may have antineoplastic potential [20-22]. While epidemiological evidence on statin use and cancer survival are as yet inconclusive $[23,24]$, some preclinical trials of mevalonate pathway inhibitors have shown promising effects as primary or adjuvant therapy in several malignancies, including ovarian cancer $[25,26]$, breast cancer [22,27], and renal cell carcinoma [28]. Despite these promising developments, clinical trials have not yet demonstrated consistently significant benefits for patients treated with mevalonate pathway inhibitors [29]. As a result, the potential of mevalonate pathway inhibitors as cancer therapy is yet unclear and remains an area of intense research interest. Our results raise the possibility that selecting patients for mevalonate inhibitor trials based on specific biomarkers of tumour phenotype (fatty macroscopic appearance, an abundance of adipophilinexpressing lipid vacuoles and HMG CoA reductase expression) may lead to more promising outcomes.

Our observations are limited to 1 case, and further studies are needed to fully interrogate lipid metabolic pathways in UM by multi-omic methods. In addition to the mevalonate pathway, futures studies would need to include glycerol phosphate, phospholipid, and eicosanoid lipid metabolic pathways. The patient in the current case was relatively young, obese, and diabetic, raising interesting questions about the effect of a lipogenic and obesogenic metabolic profile in the individual and its effect on UM development and biological behaviour. Future studies of UM are thus needed to examine sterol regulatory elementbinding proteins, a family of transcription factors that are crucial for maintaining cellular lipid homeostasis (Bengoechea-Alonso and Ericsson, 2007). Aberrant activation of sterol regulatory element-binding proteins can contribute to obesity, fatty liver disease, and insulin resistance, and could also be involved in cancer development.
In conclusion, we demonstrate HMG-CoA reductase expression in a lipid-producing high-risk UM. Although lipid production likely confers a survival (and possibly metastatic) advantage, it does not appear to be associated with unique molecular cytogenetic or cancer-associated mutations. Our observations suggest statins deserve further investigation as adjuvant therapy in high-risk $\mathrm{UM}$ and that patient selection based on tumour biomarkers of cholesterol synthesis may impact the success of therapeutic trials.

\section{Acknowledgement}

We thank Gary Gracie for performing the immunohistochemistry. The macrophotography methods were adapted from those developed by Dr. R.C. Eagle and Dr. C.A. Curcio.

\section{Statement of Ethics}

Written informed consent was obtained from the patient for the use of his data for research and publication. This study is in compliance with the Declaration of Helsinki and was approved by the institutional committee on human research at St Vincent's Hospital (HREC Ref. 17-236).

\section{Conflict of Interest Statement}

The authors have no conflicts of interest to declare.

\section{Funding Sources}

D.V.L. is supported by the Royal College of Pathologists Australasia (RCPA Scholarship in Pathology)

\section{Author Contributions}

D.V.L., D.W., R.M.C., L.H., and S.C. conceived the study. D.V.L., D.W., R.M.C., L.H., R.L., and S.C. designed the experiments. D.V.L., D.W., M.G., R.M.C., S.L., R.L., L.H., and S.C. conducted experimentation and analysed the data. D.V.L., D.W., M.G., R.M.C., S.L., R.L., L.-A.L., L.H., and S.C. wrote and contributed to the manuscript.

References

1 Amaro A, Gangemi R, Piaggio F, Angelini G, Barisione G, Ferrini S, et al. The biology of uveal melanoma. Cancer Metastasis Rev. 2017 Mar;36(1):109-40.

2 Aronow ME, Topham AK, Singh AD. Uveal Melanoma: 5-Year Update on Incidence, Treatment, and Survival (SEER 1973-2013). Ocul Oncol Pathol. 2018 Apr;4(3):145-51.

Van Ly/Wang/Conway/Giblin/Liang/ Lukeis/Lim/Hesson/Cherepanoff 
3 Virgili G, Gatta G, Ciccolallo L, Capocaccia R, Biggeri A, Crocetti E, et al.; EUROCARE Working Group. Incidence of uveal melanoma in Europe. Ophthalmology. 2007 Dec; 114(12):2309-15.

4 Baenke F, Peck B, Miess H, Schulze A. Hooked on fat: the role of lipid synthesis in cancer metabolism and tumour development. Dis Model Mech. 2013 Nov;6(6):1353-63.

5 Yavuzyigitoglu S, Kilic E, Vaarwater J, de Klein A, Paridaens D, Verdijk RM; Rotterdam Ocular Melanoma Study Group (ROMS). Lipomatous Change in Uveal Melanoma: Histopathological, Immunohistochemical and Cytogenetic Analysis. Ocul Oncol Pathol. 2016 Apr;2(3):133-5.

6 Fiorentzis M, Kalirai H, Katopodis P, Coupland SE. Adipophilin expression in primary and metastatic uveal melanoma: a pilot study. Graefes Arch Klin Exp Ophthalmol. 2017 May;255(5):1049-51.

7 Mayrhofer M, Viklund B, Isaksson A. Rawcopy: improved copy number analysis with Affymetrix arrays. Sci Rep. 2016 Oct;6(1): 36158

8 Richards S, Aziz N, Bale S, Bick D, Das S, Gastier-Foster J, et al.; ACMG Laboratory Quality Assurance Committee. Standards and guidelines for the interpretation of sequence variants: a joint consensus recommendation of the American College of Medical Genetics and Genomics and the Association for Molecular Pathology. Genet Med. 2015 May; 17(5):405-24.

9 Li MM, Datto M, Duncavage EJ, Kulkarni S, Lindeman NI, Roy S, et al. Standards and Guidelines for the Interpretation and Reporting of Sequence Variants in Cancer: A Joint Consensus Recommendation of the Association for Molecular Pathology, American Society of Clinical Oncology, and College of American Pathologists. J Mol Diagn. 2017 Jan;19(1):4-23.

10 Robertson AG, Shih J, Yau C, Gibb EA, Oba $\mathrm{J}$, Mungall KL, et al. Integrative analysis identifies four molecular and clinical subsets in uveal melanoma. Cancer cell. 2017 Aug;32(2): 204-20.e15.
11 Bakhoum MF, Esmaeli B. Molecular Characteristics of Uveal Melanoma: Insights from the Cancer Genome Atlas (TCGA) Project. Cancers (Basel). 2019 Jul;11(8):1061.

12 Vander Heiden MG, Cantley LC, Thompson CB. Understanding the Warburg effect: the metabolic requirements of cell proliferation. Science. 2009 May;324(5930):1029-33.

13 Fischer GM, Vashisht Gopal YN, McQuade JL, Peng W, DeBerardinis RJ, Davies MA. Metabolic strategies of melanoma cells: Mechanisms, interactions with the tumor microenvironment, and therapeutic implications. Pigment Cell Melanoma Res. 2018 Jan; 31(1):11-30.

14 Fujimoto M, Matsuzaki I, Yamamoto Y, Yoshizawa A, Warigaya K, Iwahashi Y, et al. Adipophilin expression in cutaneous malignant melanoma. J Cutan Pathol. 2017 Mar;44(3): 228-36.

15 Drabarek W, Yavuzyigitoglu S, Obulkasim A, van Riet J, Smit KN, van Poppelen NM, et al. Multi-Modality Analysis Improves Survival Prediction in Enucleated Uveal Melanoma Patients. Invest Ophthalmol Vis Sci. 2019 Aug;60(10):3595-605.

16 Vichitvejpaisal P, Dalvin LA, Mazloumi M, Ewens KG, Ganguly A, Shields CL. Genetic Analysis of Uveal Melanoma in 658 Patients Using the Cancer Genome Atlas Classification of Uveal Melanoma as A, B, C, and D. Ophthalmology. 2019 Oct;126(10):1445-53.

17 Gruenbacher G, Thurnher M. Mevalonate metabolism in cancer. Cancer Lett. 2015 Jan; 356(2 2 Pt A):192-6.

18 Gruenbacher G, Thurnher M. Mevalonate Metabolism in Cancer Stemness and Trained Immunity. Front Oncol. 2018 Sep;8:394.

19 Hager MH, Solomon KR, Freeman MR. The role of cholesterol in prostate cancer. Curr Opin Clin Nutr Metab Care. 2006 Jul;9(4): 379-85. DOI: 10.1097/01.mco.0000232896. 66791.62.

20 Yeganeh B, Wiechec E, Ande SR, Sharma P, Moghadam AR, Post M, et al. Targeting the mevalonate cascade as a new therapeutic approach in heart disease, cancer and pulmonary disease. Pharmacol Ther. 2014 Jul; 143(1):87-110.
21 Likus W, Siemianowicz K, Bieńk K, Pakuła M, Pathak H, Dutta C, et al. Could drugs inhibiting the mevalonate pathway also target cancer stem cells? Drug Resist Updat. 2016 Mar;25: 13-25.

22 Koohestanimobarhan S, Salami S, Imeni V, Mohammadi Z, Bayat O. Lipophilic statins antagonistically alter the major epithelial-tomesenchymal transition signaling pathways in breast cancer stem-like cells via inhibition of the mevalonate pathway. J Cell Biochem. 2018 Sep.

23 Gaist D, Hallas J, Friis S, Hansen S, Sørensen HT. Statin use and survival following glioblastoma multiforme. Cancer Epidemiol. 2014 Dec;38(6):722-7.

24 Gray RT, Loughrey MB, Bankhead P, Cardwell CR, McQuaid S, O’Neill RF, et al. Statin use, candidate mevalonate pathway biomarkers, and colon cancer survival in a population-based cohort study. Br J Cancer. 2017 Jun;116(12):1652-9.

25 Kobayashi Y, Kashima H, Wu RC, Jung JG, Kuan JC, Gu J, et al. Mevalonate Pathway Antagonist Suppresses Formation of Serous Tubal Intraepithelial Carcinoma and Ovarian Carcinoma in Mouse Models. Clin Cancer Res. 2015 Oct;21(20):4652-62.

26 Kobayashi Y, Kashima H, Rahmanto YS, Banno K, Yu Y, Matoba Y, et al. Drug repositioning of mevalonate pathway inhibitors as antitumor agents for ovarian cancer. Oncotarget. 2017 Aug;8(42):72147-56.

27 Göbel A, Thiele S, Browne AJ, Rauner M, Zinna VM, Hofbauer LC, et al. Combined inhibition of the mevalonate pathway with statins and zoledronic acid potentiates their anti-tumor effects in human breast cancer cells. Cancer Lett. 2016 May;375(1):162-71.

28 Woschek M, Kneip N, Jurida K, Marzi I, Relja B. Simvastatin Reduces Cancerogenic Potential of Renal Cancer Cells via Geranylgeranyl Pyrophosphate and Mevalonate Pathway. Nutr Cancer. 2016;68(3):420-7.

29 Iannelli F, Lombardi R, Milone MR, Pucci B, De Rienzo S, Budillon A, et al. Targeting Mevalonate Pathway in Cancer Treatment: repurposing of Statins. Recent Patents Anticancer Drug Discov. 2018;13(2):184-200. 